\title{
Solar-Thermal Storage and Room-Temperature Fast Release Using a Uniform Flexible Azobenzene-Grafted Polynorborene Film Enhanced by Stretching
}

Linxia Fu $u^{\dagger}$, Jixing Yang ${ }^{\dagger}$, Liqi Dong ${ }^{\dagger}$, Huitao Yu ${ }^{\dagger}$, Qinghai Yan ${ }^{\dagger}$, Fulai Zhao ${ }^{\dagger}$, Fei Zhai ${ }^{\dagger}$, Yunhua Xu $u^{\dagger}$, Yanfeng Dang ${ }^{\S}$, Wenping $H u^{\S}$, Yiyu Feng ${ }^{* \dagger \dagger}$, Wei Feng ${ }^{* \dagger+}+\|$

†School of Materials Science and Engineering, Tianjin University, Tianjin 300072, P. R. China.

†ianjin Key Laboratory of Composite and Functional Materials, Key Laboratory of Advanced Ceramics and Machining Technology, Ministry of Education, Tianjin University, Tianjin 300072, P. R. China.

\$School of Science, Tianjin University, Tianjin 300072, P. R. China.

IIICollaborative Innovation Center of Chemical Science and Engineering, Tianjin 300072, P.

R. China.

\section{Corresponding Author}

*Wei Feng; e-mail weifeng@tju.edu.cn.

*Yiyu Feng; e-mail fengyiyu@tju.edu.cn. 


\section{Experimental Section}

Materials: Unless otherwise noted, the reagents were obtained from commercial sources and used without further purification. All other chemicals were purchased from Sigma-Aldrich, USA.
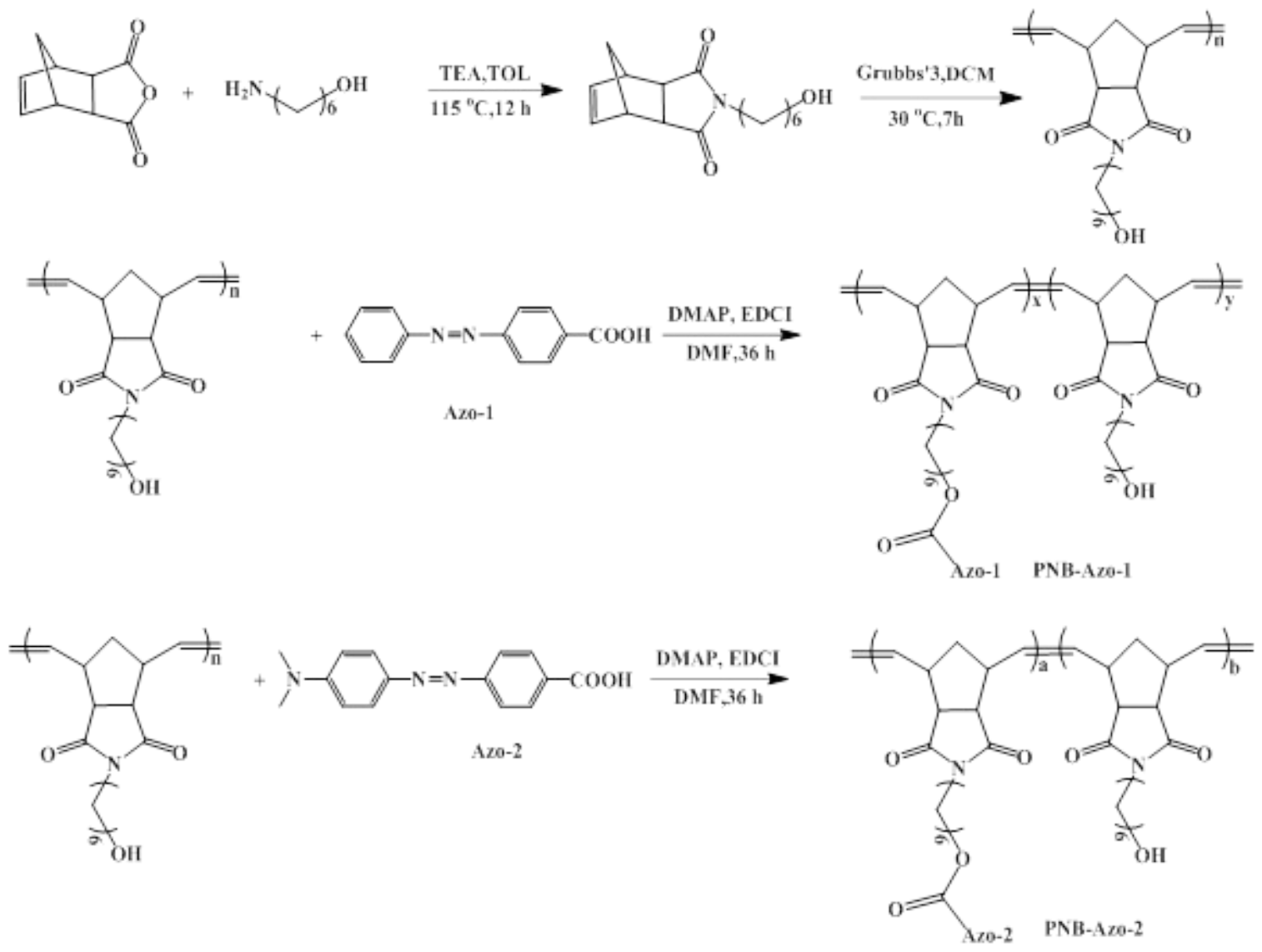

Figure S1. The synthetic route of PNB-Azo 


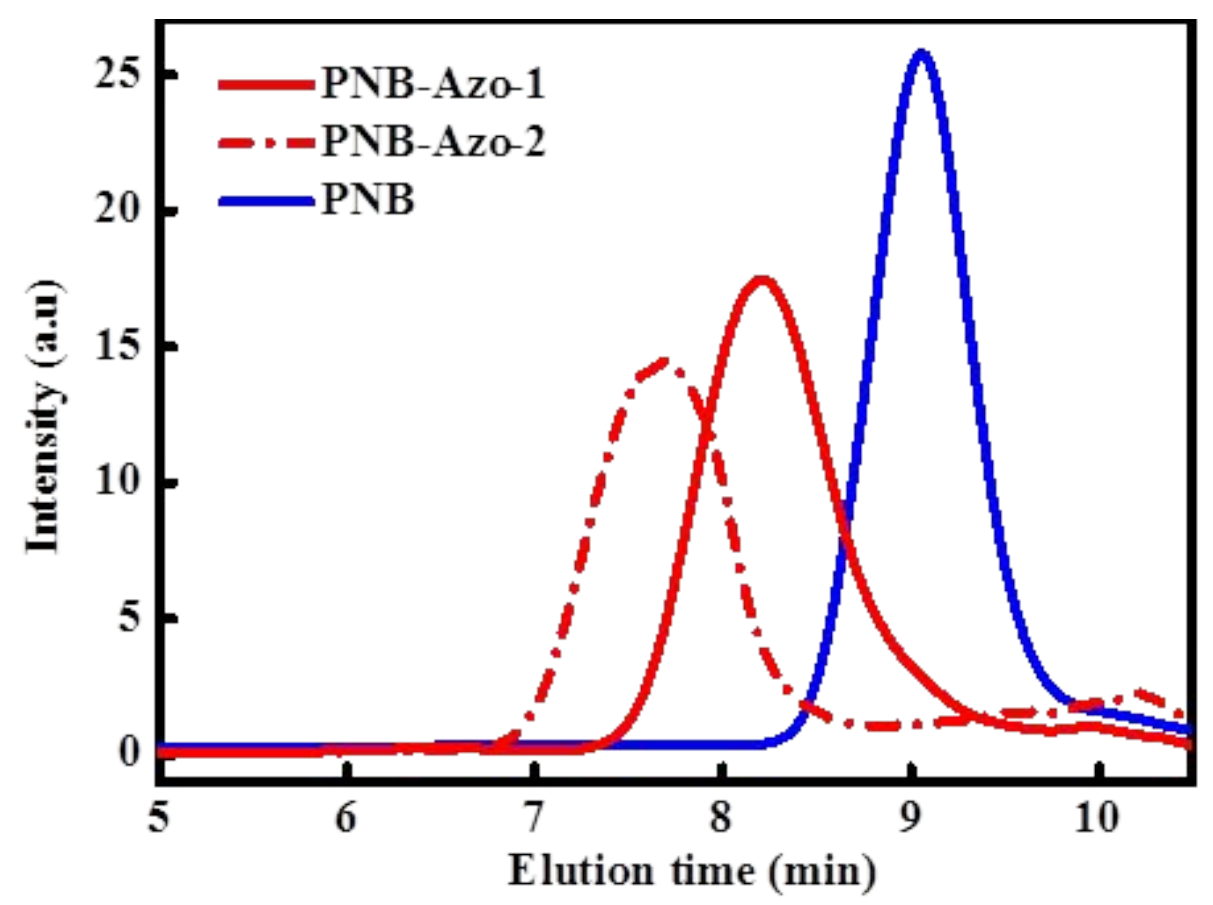

Figure S2. GPC of PNB, PNB-Azo-1 and PNB-Azo-2 

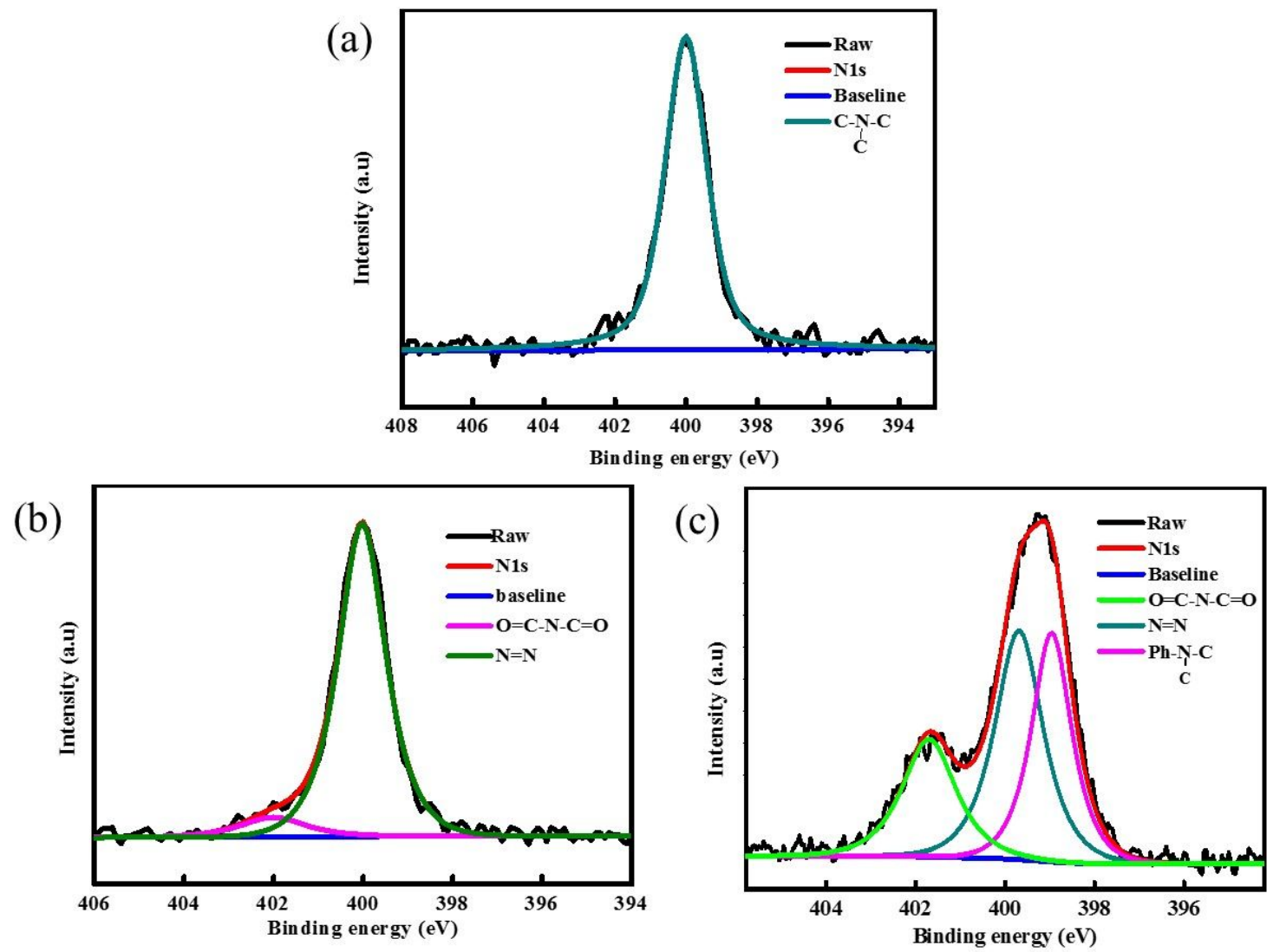

Figure S3. N1 XPS of (a) PNB, (b) PNB-Azo-1 and (c) PNB-Azo-2 


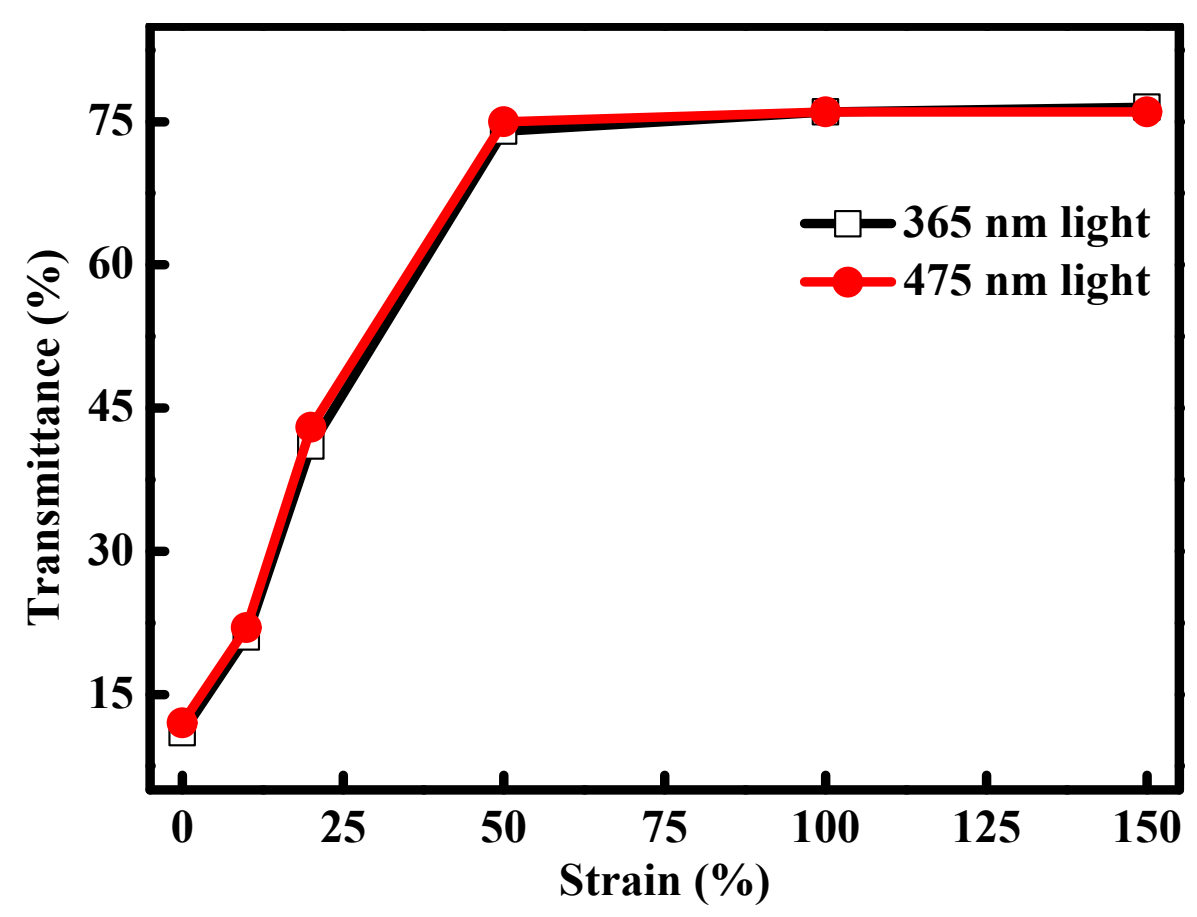

Figure S4. Transmittance of PNB-Azo-1 at $365 \mathrm{~nm}$ and $475 \mathrm{~nm}$ with the strain of $10 \%$, $20 \%, 50 \%, 100 \%$, and $150 \%$ 


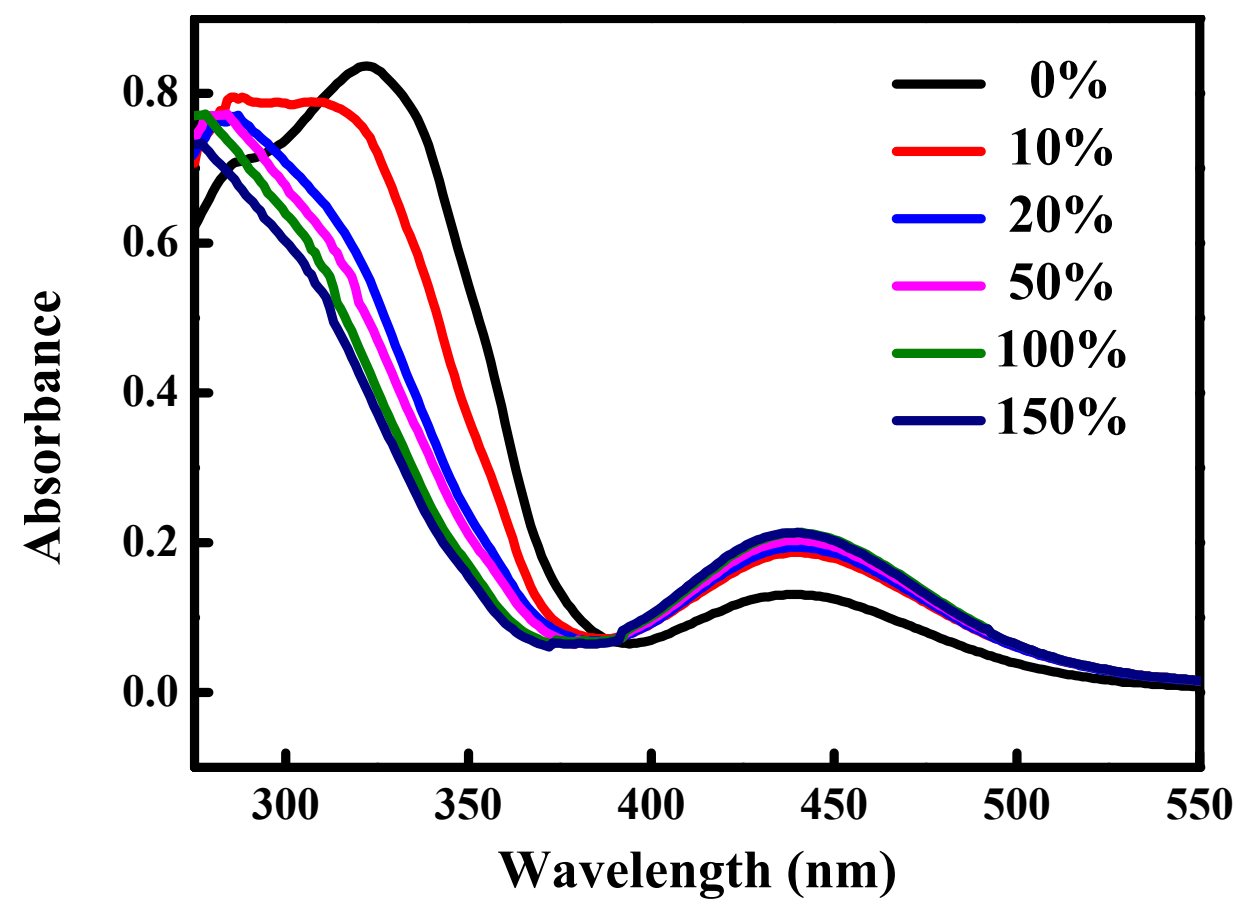

Figure S5. Absorption spectra of PNB-Azo-1 film at photoisomerization equilibrium with the strain of $10 \%, 20 \%, 50 \%, 100 \%$, and $150 \%$ 


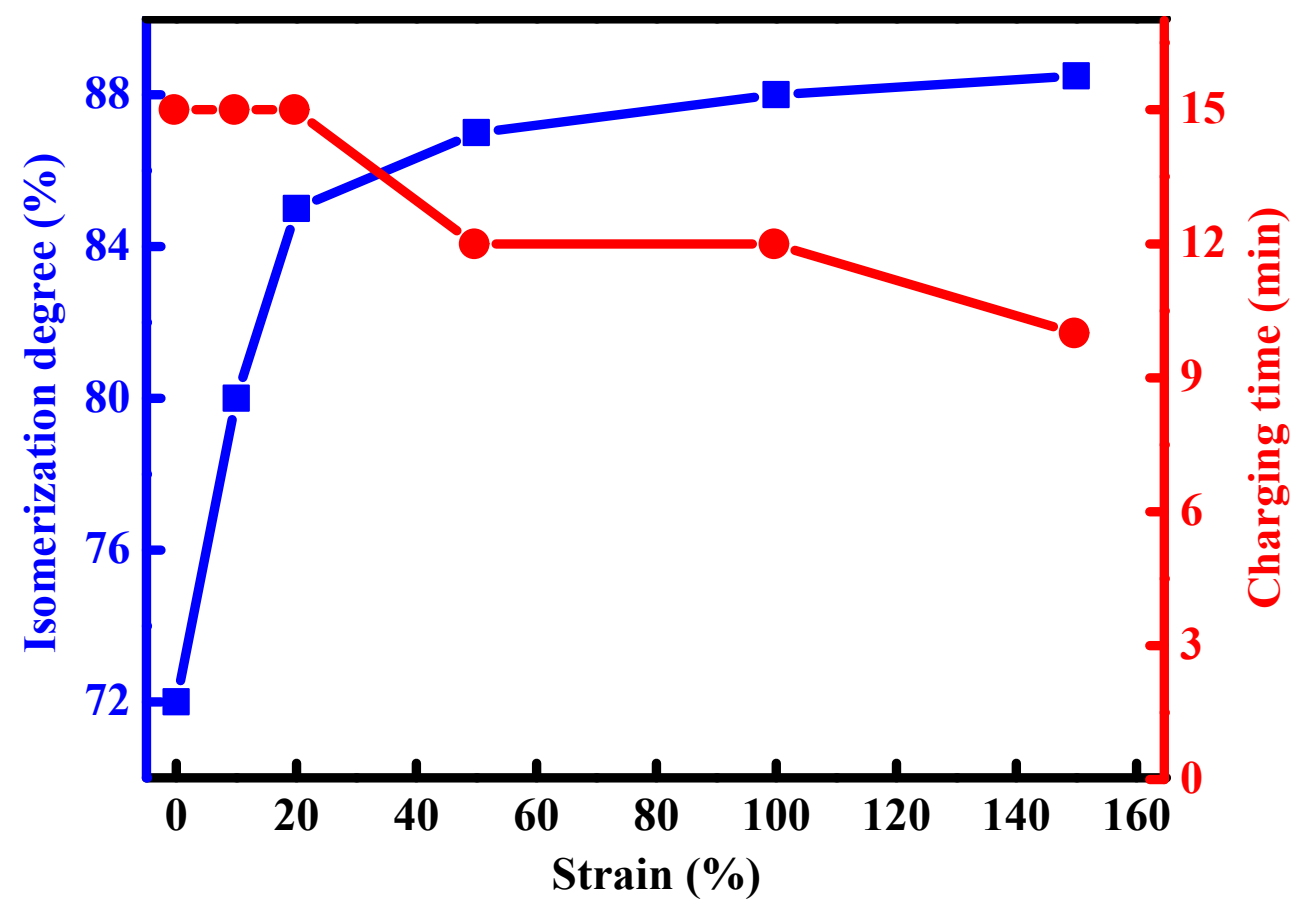

Figure S6. Isomerization degree and charging time of PNB-Azo-1 film with strain of 10\%, $20 \%, 50 \%, 100 \%$, and $150 \%$ 

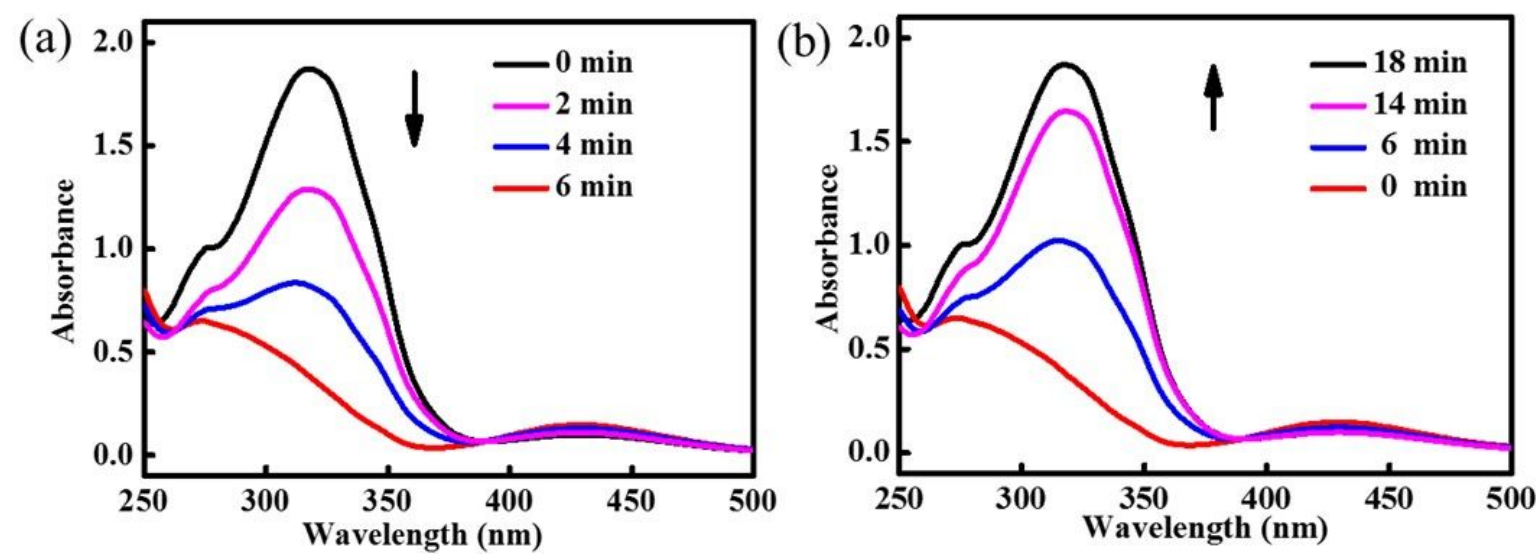

Figure S7. Time-evolved UV-vis absorption spectra of PNB-Azo-1 solution in DMF

$\left(1 \mathrm{mg} \cdot \mathrm{mL}^{-1}\right)$ (a) irradiated by UV-light $\left(365 \mathrm{~nm}, 20 \mathrm{~mW} \cdot \mathrm{cm}^{-2}\right)$ and (b) in dark after the irradiation 


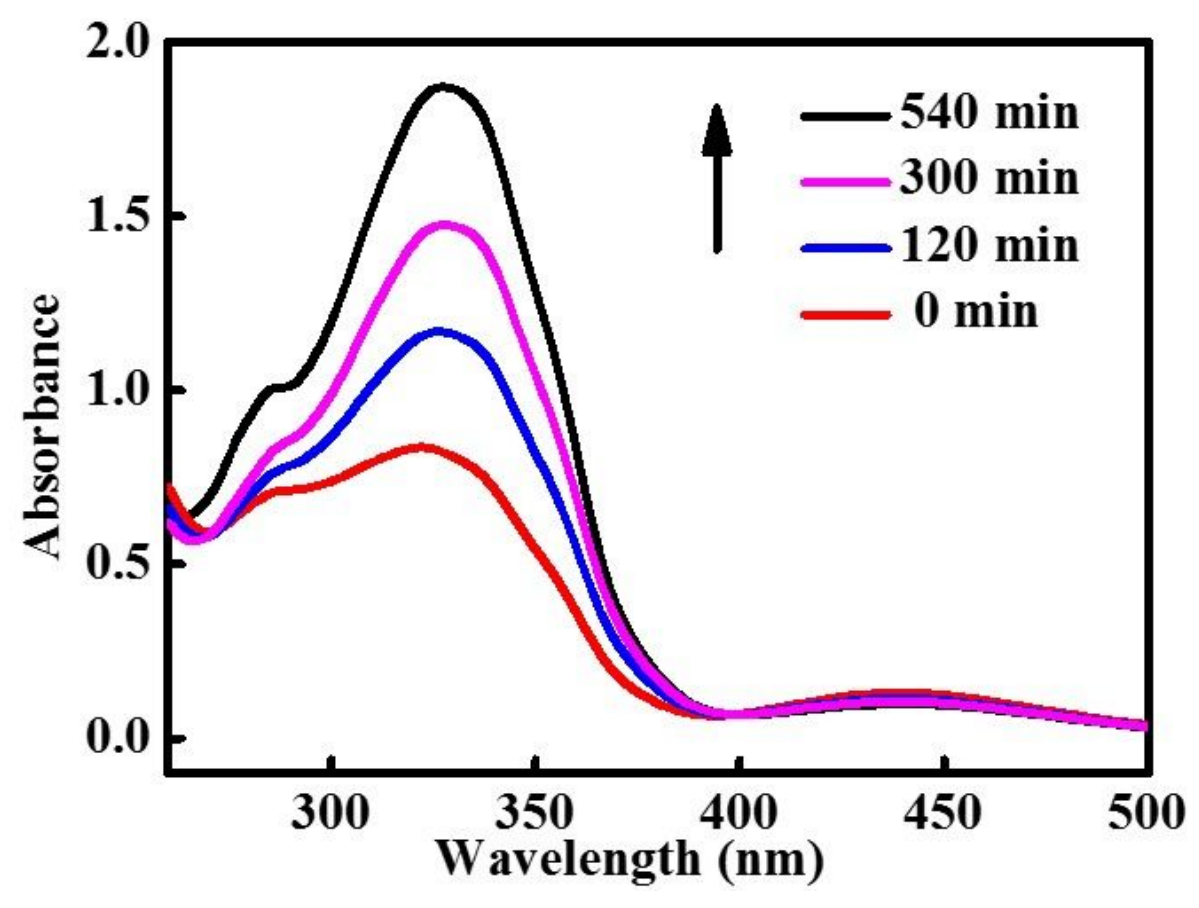

Figure S8. Time-evolved UV-vis absorption spectra of PNB-Azo-1 solution

$\left(1 \mathrm{mg} \cdot \mathrm{mL}^{-1}\right)$ in dark after the UV irradiation followed by dissolving it in DMF 

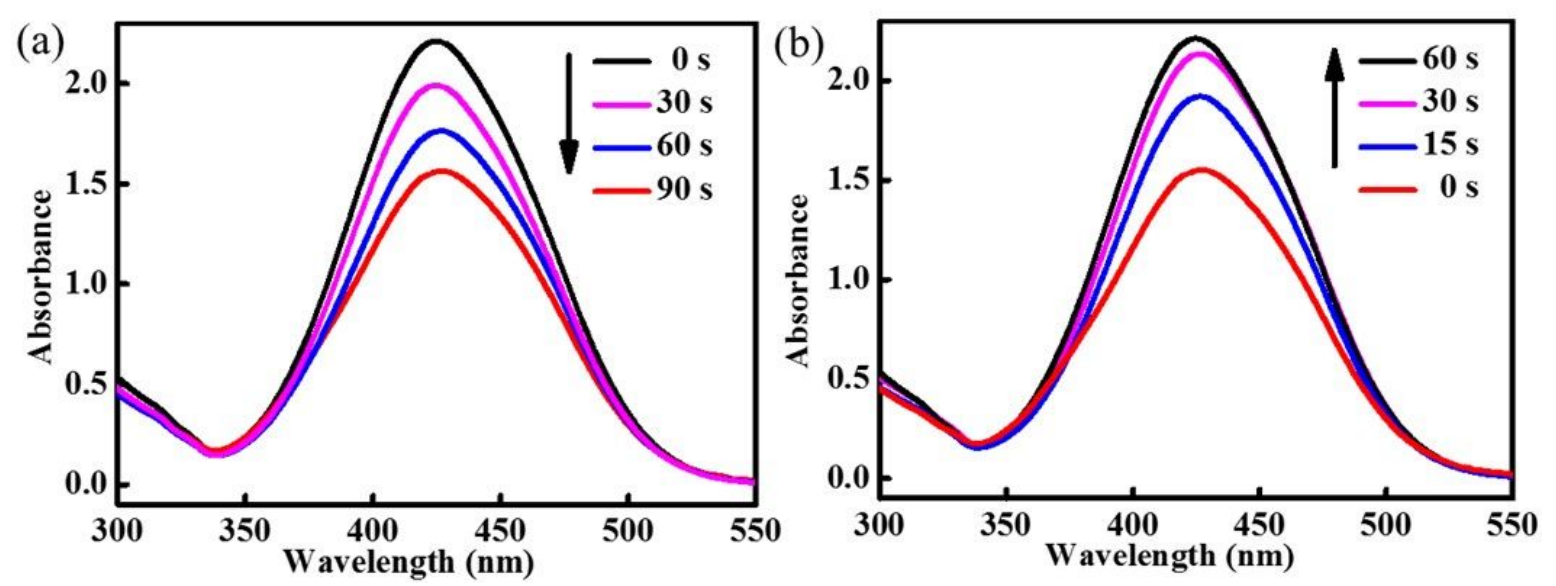

Figure S9. Time-evolved UV-vis absorption spectra of PNB-Azo-2 solution in DMF

$\left(1 \mathrm{mg} \cdot \mathrm{mL}^{-1}\right)$ (a) irradiated by UV-light $\left(365 \mathrm{~nm}, 20 \mathrm{~mW} \cdot \mathrm{cm}^{-2}\right)$ and (b) in dark after the irradiation 


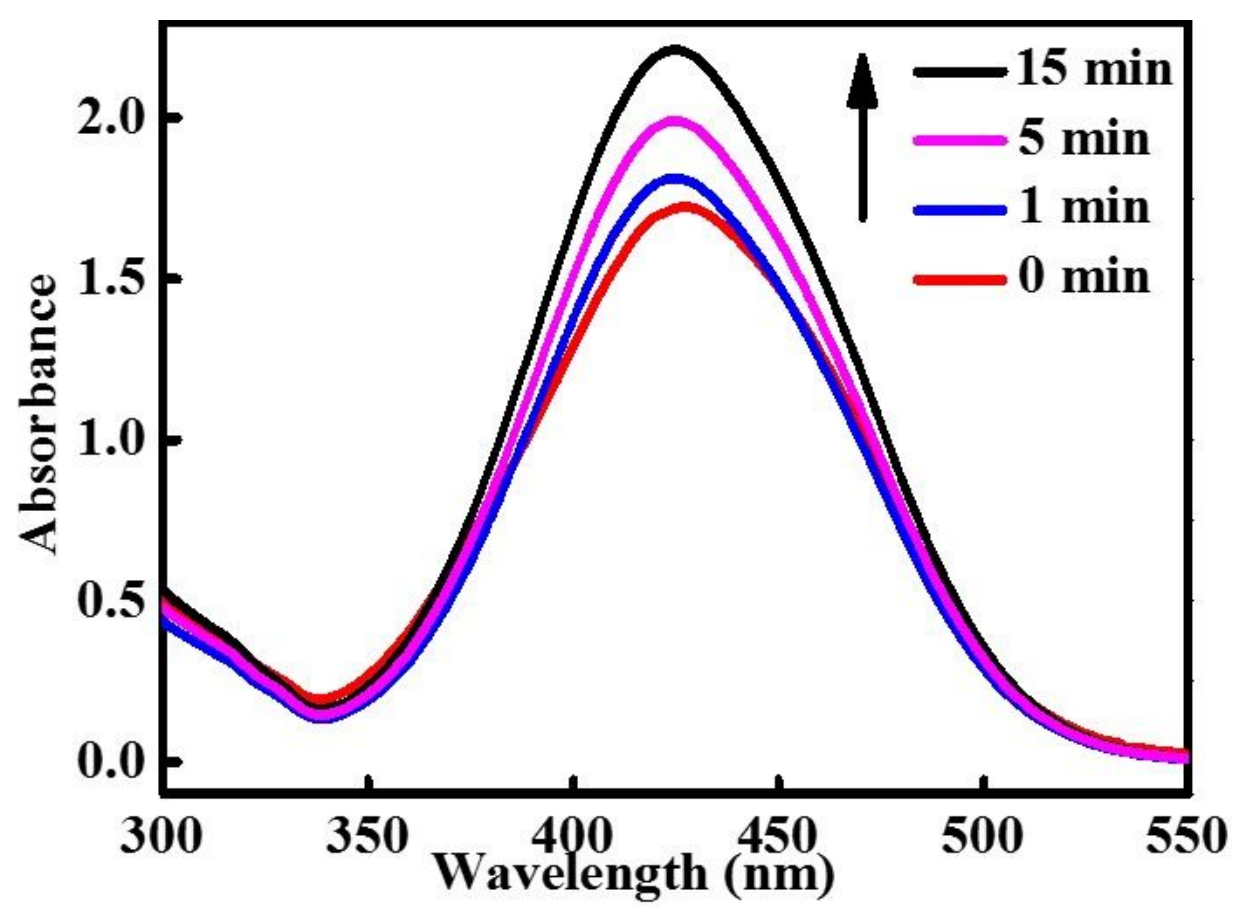

Figure S10. Time-evolved UV-vis absorption spectra of PNB-Azo-2 solution $\left(1 \mathrm{mg} \cdot \mathrm{mL}^{-1}\right)$ in dark after UV irradiation followed by dissolving it in DMF 


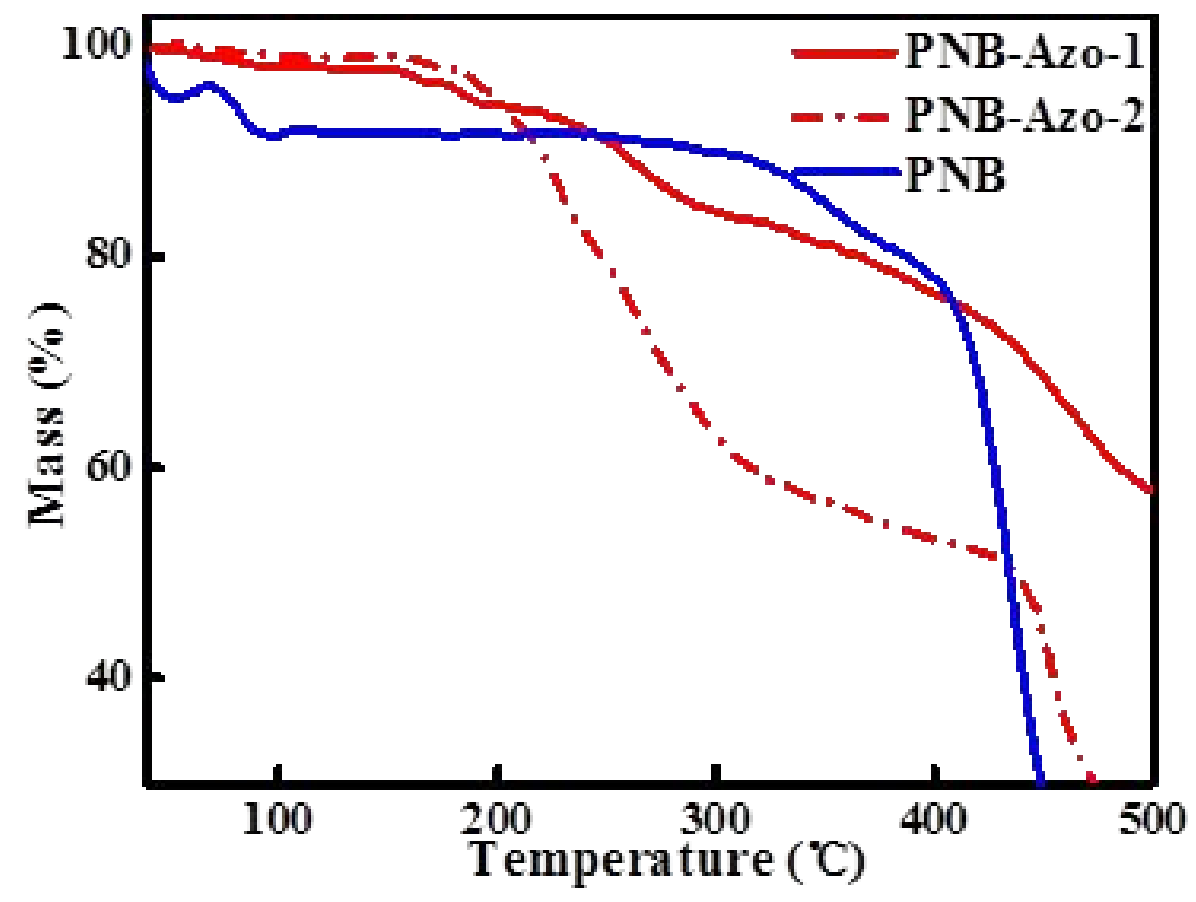

Figure S11. TGA curves of PNB, PNB-Azo-1 and PNB-Azo-2 under nitrogen at a heating rate of $5^{\circ} \mathrm{C} \cdot \mathrm{min}^{-1}$ 


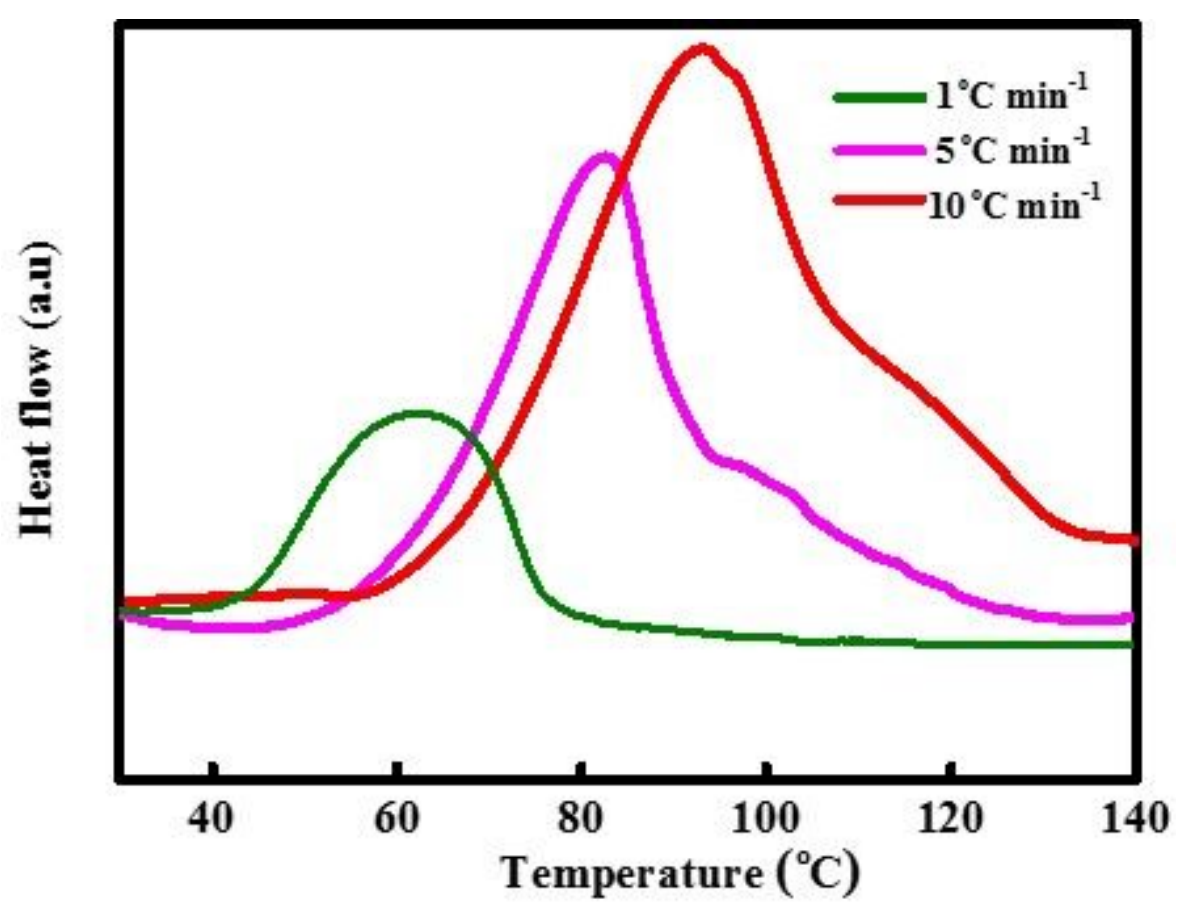

Figure S12. DSC curves of PNB-Azo- 1 film at $1{ }^{\circ} \mathrm{C}, 5^{\circ} \mathrm{C}$ and $10^{\circ} \mathrm{C} \cdot \mathrm{min}^{-1}$ heating rates 


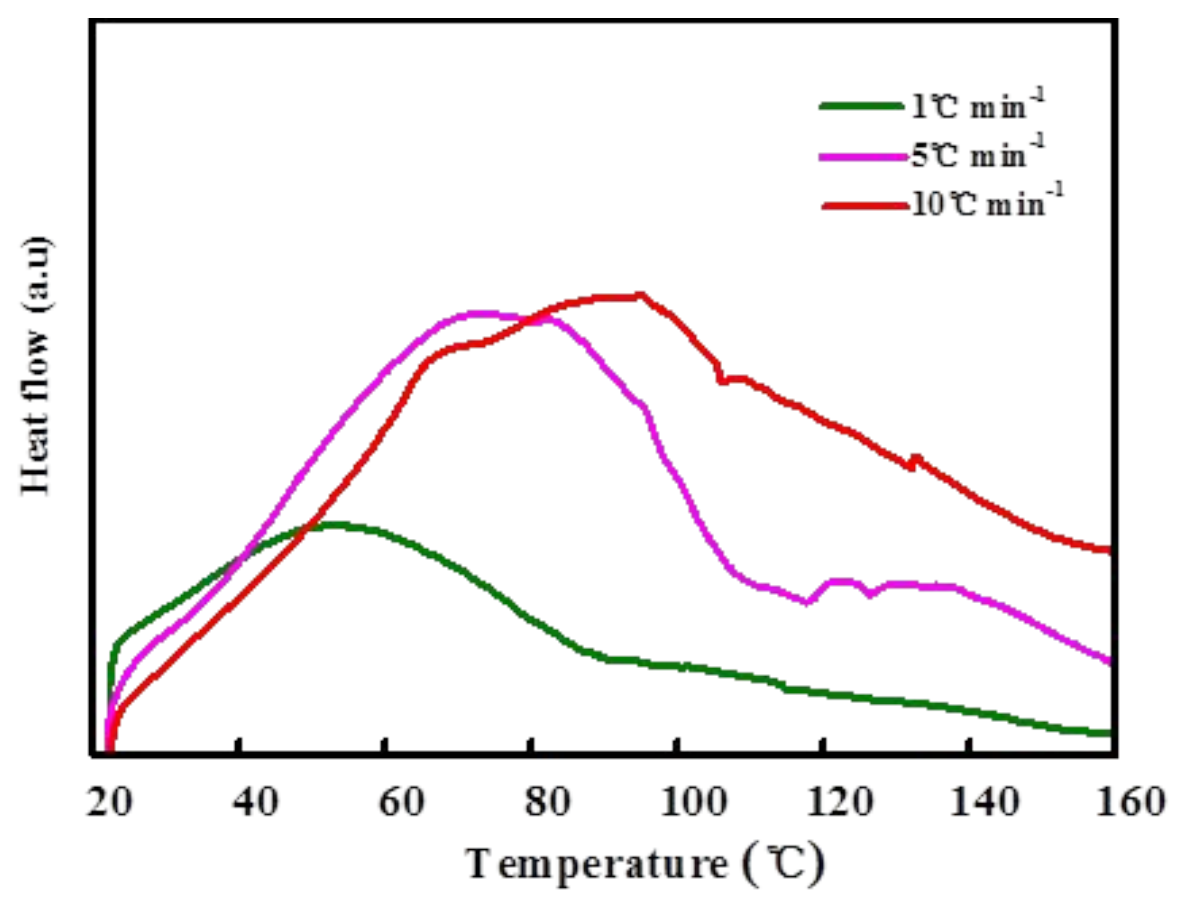

Figure S13. DSC results of PNB-Azo- $1 \mathrm{~s}-$ film at $1{ }^{\circ} \mathrm{C}, 5^{\circ} \mathrm{C}$ and $10^{\circ} \mathrm{C} \cdot \mathrm{min}^{-1}$ heating rates 


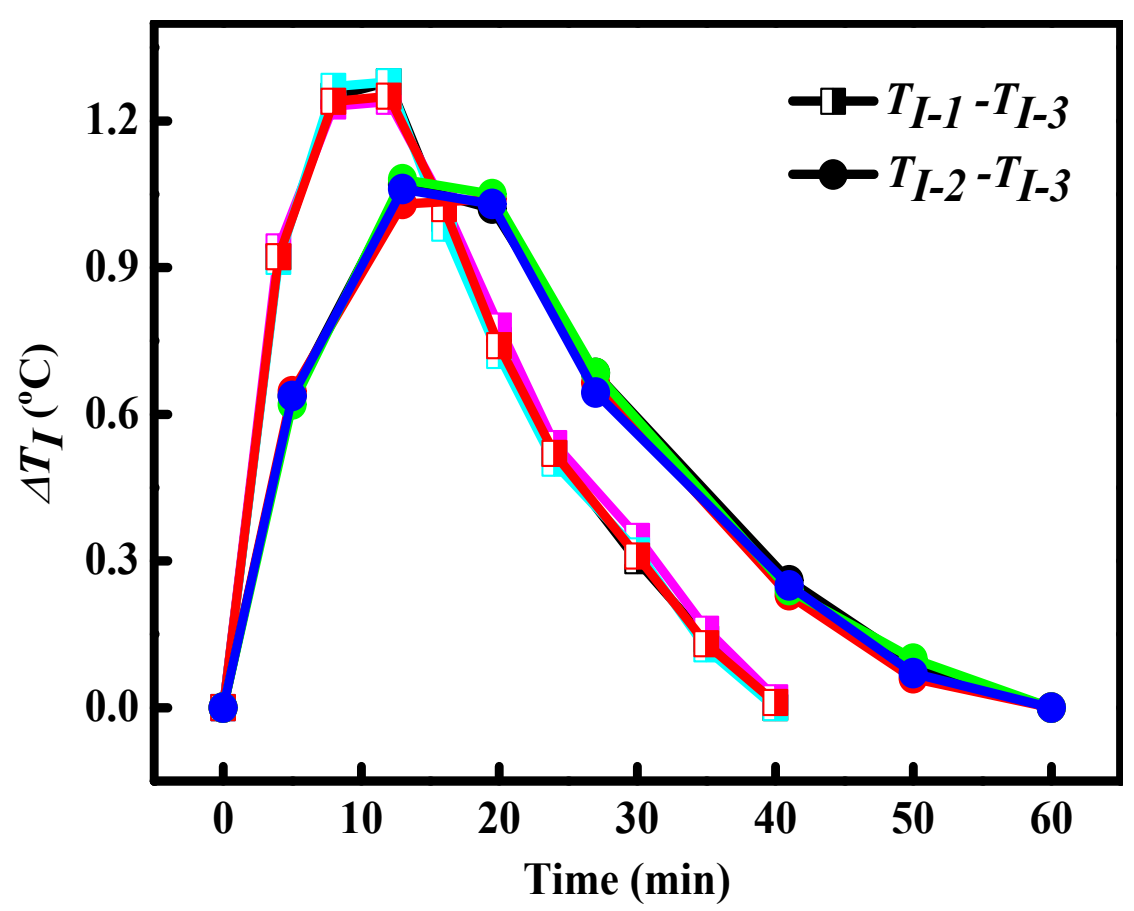

Figure S14. $\Delta T_{I}$ at different times of PNB-Azo film for four samples in static state under blue-light irradiation at room temperature 


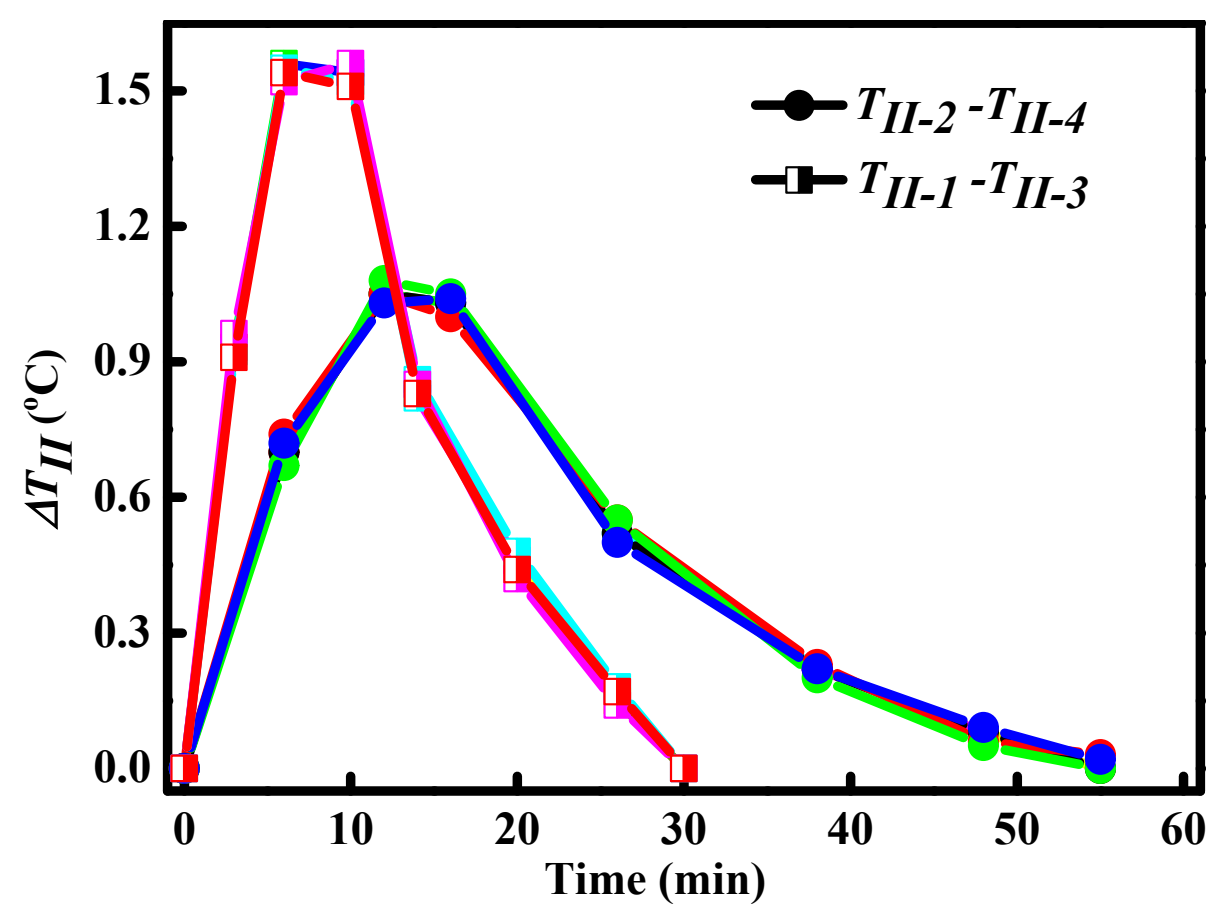

Figure S15. $\Delta T_{I I}$ at different times of PNB-Azo film for four samples in dynamic state under blue-light irradiation at room temperature 
Table S1. Chemical element of PNB and PNB-Azo and its corresponding grafting density

\begin{tabular}{cccc} 
& Atomic percent of element & $\boldsymbol{G}_{\boldsymbol{d}}(\mathrm{mol} \mathrm{\%})$ \\
Name & $\mathrm{C}$ & $\mathrm{N}$ & \\
\hline PNB & 95.8 & 4.2 & -- \\
PNB-Azo-1 & 93.5 & & 48.5 \\
PNB-Azo-2 & 92.4 & 6.5 & 43.1 \\
\hline
\end{tabular}

--There is no data 
Table S2. Temperature difference of PNB-Azo-1 hand induced by heat release at different times after static stretching

\begin{tabular}{llllllllllll}
\hline \multicolumn{1}{c}{ PNB-Azo-1 s-film } & \multicolumn{10}{c}{ PNB-Azo-1 film } \\
\hline Time & $\Delta \boldsymbol{T}_{\boldsymbol{1}}$ & $\Delta \boldsymbol{T}_{2}$ & $\Delta \boldsymbol{T}_{3}$ & $\Delta \boldsymbol{T}_{4}$ & Ave. & Time & $\Delta \boldsymbol{T}_{\boldsymbol{I}}$ & $\Delta \boldsymbol{T}_{2}$ & $\Delta \boldsymbol{T}_{3}$ & $\Delta \boldsymbol{T}_{4}$ & Ave. \\
$(\mathrm{min})$ & $\left({ }^{\circ} \mathrm{C}\right)$ & $\left({ }^{\circ} \mathrm{C}\right)$ & $\left({ }^{\circ} \mathrm{C}\right)$ & $\left({ }^{\circ} \mathrm{C}\right)$ & $\left({ }^{\circ} \mathrm{C}\right)$ & $(\mathrm{min})$ & $\left({ }^{\circ} \mathrm{C}\right)$ & $\left({ }^{\circ} \mathrm{C}\right)$ & $\left({ }^{\circ} \mathrm{C}\right)$ & $\left({ }^{\circ} \mathrm{C}\right)$ & $\left({ }^{\circ} \mathrm{C}\right)$ \\
0 & 0 & 0 & 0 & 0 & 0 & 0 & 0 & 0 & 0 & 0 & 0 \\
4 & 0.92 & 0.94 & 0.91 & 0.91 & 0.92 & 4 & 0.51 & 0.48 & 0.47 & 0.5 & 0.64 \\
8 & 1.27 & 1.28 & 1.2 & 1.25 & 1.25 & 12 & 1.09 & 1.07 & 1.04 & 1.08 & 1.07 \\
12 & 1.26 & 1.29 & 1.28 & 1.29 & 1.28 & 20 & 1.06 & 1.03 & 0.98 & 0.99 & 1.02 \\
20 & 0.75 & 0.76 & 0.75 & 0.74 & 0.75 & 30 & 0.62 & 0.61 & 0.58 & 0.63 & 0.68 \\
24 & 0.52 & 0.54 & 0.51 & 0.53 & 0.52 & 40 & 0.31 & 0.28 & 0.29 & 0.27 & 0.26 \\
30 & 0.28 & 0.31 & 0.29 & 0.3 & 0.3 & 50 & 0.11 & 0.08 & 0.07 & 0.1 & 0.09 \\
40 & 0 & 0 & 0 & 0 & 0 & 60 & 0.03 & 0 & 0 & 0 & 0 \\
\hline
\end{tabular}


Table S3. Temperature difference of PNB-Azo-1 finger induced by heat release at different times under dynamic stretching

\begin{tabular}{|c|c|c|c|c|c|c|c|c|c|c|c|}
\hline \multirow[b]{2}{*}{$\begin{array}{l}\text { Time } \\
(\mathrm{min})\end{array}$} & \multicolumn{4}{|c|}{ PNB-Azo-1 s-film } & \multicolumn{7}{|c|}{ PNB-Azo-1 film } \\
\hline & $\begin{array}{l}\Delta T_{1} \\
\left({ }^{\circ} \mathrm{C}\right)\end{array}$ & $\begin{array}{l}\Delta T_{2} \\
\left({ }^{\circ} \mathrm{C}\right)\end{array}$ & $\begin{array}{l}\Delta T_{3} \\
\left({ }^{\circ} \mathrm{C}\right)\end{array}$ & $\begin{array}{l}\Delta T_{4} \\
\left({ }^{\circ} \mathrm{C}\right)\end{array}$ & $\begin{array}{l}\text { Ave. } \\
\left({ }^{\circ} \mathrm{C}\right)\end{array}$ & $\begin{array}{l}\text { Time } \\
(\min )\end{array}$ & $\begin{array}{l}\Delta T_{1} \\
\left({ }^{\circ} \mathrm{C}\right)\end{array}$ & $\begin{array}{l}\Delta T_{2} \\
\left({ }^{\circ} \mathrm{C}\right)\end{array}$ & $\begin{array}{l}\Delta \boldsymbol{T}_{3} \\
\left({ }^{\circ} \mathrm{C}\right)\end{array}$ & $\begin{array}{l}\Delta \boldsymbol{T}_{4} \\
\left({ }^{\circ} \mathrm{C}\right)\end{array}$ & $\begin{array}{l}\text { Ave. } \\
\left({ }^{\circ} \mathrm{C}\right)\end{array}$ \\
\hline 0 & 0 & 0.02 & 0 & & 0 & 0 & 0 & 0.01 & 0 & 0 & 0 \\
\hline 3 & 0.95 & 0.94 & 0.9 & 0.89 & 0.92 & 6 & 0.7 & 0.72 & 0.71 & 0.68 & 0.69 \\
\hline 6 & 1.49 & 1.53 & 1.52 & 1.54 & 1.52 & 12 & 1.05 & 1.07 & 1.04 & 1.03 & 1.05 \\
\hline 10 & 1.47 & 1.52 & 1.51 & 1.5 & 1.5 & 16 & 1.03 & 1.04 & 1.05 & 1.01 & 1.03 \\
\hline 14 & 0.84 & 0.87 & 0.86 & 0.88 & 0.85 & 26 & 0.52 & 0.54 & 0.5 & 0.53 & 0.51 \\
\hline 20 & 0.49 & 0.51 & 0.45 & 0.47 & 0.48 & 38 & 0.22 & 0.21 & 0.24 & 0.21 & 0.23 \\
\hline 26 & 0.13 & 0.18 & 0.16 & 0.19 & 0.16 & 48 & 0.08 & 0.08 & 0.06 & 0.09 & 0.1 \\
\hline 30 & 0.03 & 0.01 & 0 & 0 & 0.01 & 55 & 0 & 0.02 & 0.01 & 0 & 0 \\
\hline
\end{tabular}




\section{Equation S1}

$D_{i}=\frac{\delta_{2}-\delta_{1}}{\delta_{1}} \times 100 \%$

where $D_{i}$ is the isomerization degree and $\delta_{2}$ is peak areas of the chemical shifts of

PNB-Azo in the metastable state (cis-rich), irradiated by UV light, $\delta_{1}$ is peak areas of the chemical shifts of PNB-Azo before UV irradiation. 


\section{Equation S2}

$\ln \frac{A_{\infty}-A_{t}}{A_{\infty}-A_{0}}=-\mathrm{k}_{r} t$

$A_{0}$ is the absorption intensity of PNB-Azo in the metastable state (cis-rich) irradiated by UV light, $A_{t}$ is the absorption intensity of PNB-Azo reversing for " $t$ " time and $A_{\infty}$ is the absorption intensity of PNB-Azo after cis-trans reversion. 